\title{
viewpoint
}

\section{Useful Websites}

National Association of Science Writers: http://www.nasw.org/ Association of British Science Writers: http://www.absw.org.uk/ American Association for the Advancement of Science: http://www.aaas.org/ Science Communication courses in the UK: http://www.absw.org.uk/courses.htm Boston University Science Journalism Program: http://www.bu.edu/com/jo/ science.html

Columbia University Earth \& Environmental Science Journalism: http:// www.columbia.edu/cu/gsas/depts/eesj.html

Metcalf Institute for Marine \& Environmental Reporting, University of Rhode Island: http://www.gso.uri.edu/metcalf/main.html

University of Missouri Science Journalism Center: http://science.jour.missouri.edu/ MIT Graduate Program in Science Writing: http://web.mit.edu/sciwrite/

New York University's Science and Environmental Reporting Programme: http:// www.nyu.edu/gsas/dept/journal/serp/

Johns Hopkins University Writing Seminars: http://www.jhu.edu/ writsem/ University of Washington's Department of Technical Communication: http:// www.uwtc.washington.edu/

tell us stories that live in our minds long after we read their words.

After 20 years of training some 200 graduate students, I can report some progress in convincing editors that properly trained scientists can do the job of reporting on science for general readers. However-and this is a big howevereditors still insist that these scientists must, no matter what their qualification in science, move through some version of the traditional gauntlet of disparate beats at small and medium-sized newspapers before assuming a high-profile science writer position at a national newspaper. No matter how well grounded one is in science, there are no shortcuts to becoming a nationally prominent science reporter.

Indeed, all I can say to scientists who are considering science writing as a career is this: succeeding in science journalism is no easier than succeeding in science. One must be called to it, almost as one is called to a religious life. Our students have, in part perhaps for mystical reasons, done well. Nearly all those we have trained have moved into jobs in newspapers, magazines, museums, zoos, aquariums, and news offices in universities and government laboratories. A few have gone into the more specialised fields of technical writing or medical writing. Not a single one, interestingly, has gone back to the laboratory bench.

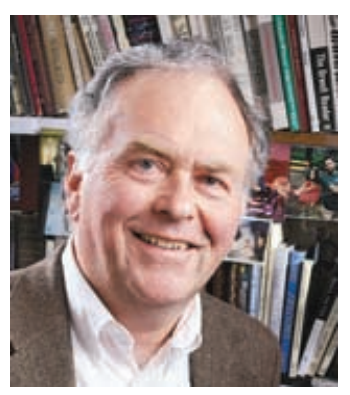

John Wilkes is Director of the Science Communication Program at the University of California, Santa Cruz, CA

E-mail: WILKES@CATS.UCSC.EDU

http://scicom.ucsc.edu

DOI: $10.1093 /$ embo-reports/kvf225

\section{Immunology at the crossroads}

\section{As decades of research have resulted in few clinical applications, it is time to think about new research strategies to understand the workings of the immune system $\bullet$ by Antonio Coutinho}

Ever since Niels Jerne introduced the Darwinian principles of 'variation and selection' into immunology in 1955, the field has truly flourished. Immunologists can now claim an impressive record; during the past 40 years, they have deciphered a significant number of intricate molecular and cellular mechanisms. Indeed, the names of many immunologists enrich the list of Nobel Prize winners, confirming that this field has been at the forefront of biological research for many decades. Nearly 30 years ago, in a lecture at the Institut Pasteur in Paris, France, Jerne estimated that the number of immunologists in the world had tripled every 20 years from the late 19th century, to reach about 10000 in 1970 (Jerne, 1974). This number is now likely to be above 40000 , and a paper in immunology is published, on average,

made a remarkable contribution to general biology. Jerne used to say that lymphocytes are the Escherichia coli of eukaryotes, and many advances in cell differentiation, the cell cycle, signalling, intracellular protein trafficking, DNA

The paradox of today's immunology is that tremendous progress in basic science has been matched by only a few clinical applications

every 15 minutes. Today, not only do we know many of the cell types, molecules and genes that protect us from diseasecausing agents, but immunology has also recombination and repair, and the regulation of gene expression owe their discovery to this extraordinary cell type. The study of the immune system has also contributed to an 


\section{viewpoint}

understanding of phenomena such as the evolution of gene complexes, the principles of cell migration and homing, homeostasis and cell turnover. And even although other biologists are often dismayed by the jargon and acronyms used by immunologists, 'the antibody problem' nevertheless fascinated the founding fathers of molecular biology, and this widespread interest in the essential problems of immunology continues to this day.

This fascination has also been triggered by the clinical implications of immunologyperhaps the very first branch of 'molecular medicine' - for there is an extraordinarily high frequency of 'immunological' conditions. Allergies and autoimmune diseases, for example, strike some 25 and $10 \%$, respectively, of the population in the developed world, and both are on the increase as societies are becoming 'cleansed' of infectious diseases (Bach, 2002). Furthermore, despite the roller-coaster of hopes and disappointments, many groups in the world continue to work on cancer immunity, convinced that vaccines

A short history of immunology
1798 Edward Jenner develops first
vaccine against smallpox
1877 Paul Ehrlich discovers mast cells
1879 Louis Pasteur develops attenu-
ated chicken colera vaccine
1883 Ilya llyich Mechnikov describes
cellular theory of vaccination
1885 Louis Pasteur develops rabies
vaccination
1900 Ehrlich's antibody formation theory
1901 Emil Adolf von Behring receives
Nobel Prize for serum therapy
against diphtheria
1906 Clemens von Pirquet
coins the term allergy
1908 Paul Ehrlich \& llya llyich Mechnikov
receive Nobel Prize

only a few successful clinical applications. Indeed, we have at present no specific and effective therapy to treat autoimmune diseases, and we continue to treat aller- and, imminently, of poliomyelitis just how powerful vaccines can be, and we are well aware of their tremendous social and economical value - at the end of the last century, nine vaccines boasted a $99 \%$ reduction in the number of relevant disease cases in the USA (Rappuoli et al., 2002). Yet, centuries after Jenner's 'vaccination' and more than 100 years after Louis Pasteur's principle of 'attenuated' vaccines, we are still totally devoid of vaccines against chronic infections and against a large number of acute infections, and several decades of 'recombinant DNA technology' and 'biotechnology' have failed to produce all the new effective, 'no-risk' vaccines we expected. Most vaccines used today are of the 'conventional' type, many of which were discovered when we had little knowledge of immunology, and absolutely no information on the cells and molecules of the immune system.

It is therefore necessary to discuss the reasons for this apparent paradox. Obviously, the lack of clinical success means that our underagainst tumour antigens will bring a clinical solution to the treatment of these diseases. Organ and tissue transplantations are also on the rise with the associated problems of graft rejection and the resulting need for immunosuppressive therapies. Current research on human and adult stem cells will also need to confront this problem-diabetic patients, for instance, will still rapidly reject their new insulin-producing cells, even if they are derived from their own stem cells. Many other groups work on the 'immunology of reproduction', trying to correct infertility or, conversely, 'vaccinate against pregnancy'. In the developing world, the main focus of immunological research is on the development of new vaccines against many viral, bacterial and parasitic agents that continue to claim millions of lives, particularly of children.

This is the paradox of today's immunology: the apparently tremendous progress in basic science has been matched by gies symptomatically, just as we did 50 years ago. The clinical development of cancer vaccines seems to have stalled, and we are unable to overcome the rejection of organ, cell or tissue grafts, subjecting transplantation patients to the consequences of long-term immunosuppressive

$$
\begin{aligned}
& \text { Most of us would agree that the } \\
& \text { unsolved problems in immunology } \\
& \text { related to the question of } \\
& \text { self-tolerance and regulation } \\
& \text { of immune responses }
\end{aligned}
$$

therapy. Furthermore, after 20 years of developing and 'humanising' monoclonal antibodies, only 10 have been approved for clinical use, although a total of 186 have entered clinical trials (Reichert, 2001). The case of vaccines against infectious diseases is particularly striking. We know from the eradication of smallpox standing of the immune system, despite decades of intense research, still remains elusive. Perhaps it is time to step back and think about what we have missed in the past and identify the new research directions and strategies that are necessary to answer the many questions that remain open. New understanding seems particularly relevant to how the immune system functions on the organismal level. Such an undertaking will not be easy, I fear, and it will require the concerted efforts of many researchers around the world, probably mirroring the scope and extent of the Human Genome Project.

But let me first underline that we have indeed scored some clinical success in treating immunodeficiencies. These range from the passive transfer of immunoglobulins to the transplantation of compatible bone marrow or hemopoietic stem cells, and, most recently, to gene therapy. In this particular case, the approach is straightforward: once the cellular, molecular or 


\section{viewpoint}

genetic defect is identified, replace it to correct the condition. Obviously, this predominantly analytical solution works fine whenever the problem involves only components, suggesting that the solution to all the other failures will require more than an analysis of single cells, genes or molecules.

Most of us would probably agree that all the unsolved problems in immunology relate, in one way or another, to the question of self-tolerance and the regulation of immune responses. Indeed, this is today's most important and controversial topic,

\section{In vitro cell culture techniques are obviously not appropriate to investigate tolerance and tend to generate misleading data}

with many good scientists on opposing sides of the debate. The field of immunology is divided between those who defend that tolerance is 'recessive' and based on the 'cell by cell' elimination or inactivation of self-recognising lymphocytes, and those who argue that tolerance is 'dominant' and maintained by 'regulatory' mechanisms controlling pathological autoimmunity and tissue rejection. While the former view has been overwhelmingly favoured over the past 40 years, the theory of 'dominant' tolerance has recently gained many supporters, including some of those who have actively defended 'clonal deletion' in the past. The problem is not just academic_-clinicians need to decide every day how to treat autoimmune patients. The conventional idea of 'recessive' tolerance suggests that autoimmune diseases result from exacerbated reactivity, and therapies based on this notion aim at killing or inhibiting lymphocytes. Current treatments are non-specific, and have been compared to using lobotomy to treat schizophrenic patients. In sharp contrast, the alternative view of 'dominant' tolerance attributes the disease to a deficit of regulatory lymphocytes, suggesting that immunosuppressive regimes may actually jeopardise 'spontaneous' remissions, and only provide symptomatic amelioration through their anti-inflammatory effects. Reflecting the basic scientific dispute, many of the clinical trials that are currently ongoing aim to either stimulate or suppress the immune system of autoimmune patients.
The present disarray in treating autoimmune diseases has been caused equally by the great expectations raised largely by the mainstream media. These promises have not been fulfilled-some approaches have already failed in early clinical trials and others are likely to befall the same fate. And the few recent cases of limited success are not based on any rational drug design or better understanding of the immune system. For example, treatment with IFN- $\beta$ helps a subsection of multiple sclerosis patients, and the administration of high doses of serum immunoglobulin G from healthy donors has resulted in astonishing improvements in some cases of inflammatory and/or autoimmune diseases, but we do not know the mechanisms underlying these beneficial effects. Yet, these two therapies boast a world-wide market of more than US\$2 billion a year!

This frustrating lack of success in treating disorders related to the immune system-with the exception of immunodeficiencies-is likely to be due to our ignorance, which is compounded by the nature of the problem and our current approaches and research strategies. Although many researchers now acknowledge that natural tolerance is acquired during development, only a few immunologists show any interest in developmental biology and in how 'tolerant' lymphocyte repertoires are established during embryonic and perinatal development. Most current papers on tolerance report experiments with adult animals, probably because there is an astonishing confusion between the development of the organism and the differentiation of lymphocytes. In proposing his clonal selection theory, Macfarlane Burnet assumed that all lymphocytes are generated during pre-natal development and postulated that tolerance is established in embryos by the elimination of autoreactive lymphocytes. When it became evident that new lymphocytes are produced throughout life, Burnet's proposal seemed useless, until Joshua Lederberg rescued it by assuming that lymphocytes pass through a 'deletionsusceptibility' period whenever they are generated during pre- or post-natal life. Lederberg thus reduced tolerance on the organismal level to the behaviour of single cells while disregarding developmental tolerance. However, immunologists know from the classical Nobel Prize experiments' of Peter Medawar that there is a time window for tolerance induction, which is limited to embryonic and perinatal life and thus pertains to the immune system's or the organism's development.

Immunologists also often forget that natural tolerance is a property of organisms, not of cells or molecules. During the past two decades or so, it has become increasingly clear that tolerant organisms invariably harbour 'non-tolerant' cells and autoantibodies. Consequently, in vitro cell culture techniques that are extremely successful for studying lymphocytes, are obviously not appropriate for investigating tolerance, and tend to generate misleading data. We therefore need new organism-centred approaches to understand how the immune system avoids the destruction of the organism in which it develops, while still protecting against infections. But such experiments consume a lot of time and money, so many researchers avoid them in the face of fierce competition to publish first. Furthermore, if natural tolerance is indeed 'dominant' and likely to be mediated by 'regulatory lymphocytes', the central mechanisms must be 'supra-clonal' and involve networks of cells and molecules. Since self-tolerance is specific and must allow for conventional immune responses to non-self antigens, such a 'toleranceregulating network' will involve V-regions of lymphocytes. This argument leads us to Jerne's last contribution to immunology in the early 1970s, namely the 'immune network' theory, which, although it attracted a lot of attention for more than a decade,

The question of how natural tolerance develops is fundamental and has an enormous clinical significance

unfortunately fell into oblivion. The notion of 'complexity' that Jerne's theory imposes on the immune system has discouraged many researchers from investigating these networks, and the apparent 'sterility' of solipsistic network approaches has chased others away. But more importantly, it was the incompatibility of Jerne's theory with the predominant idea of 'recessive tolerance' that has led most immunologists to ignore networks within the immune system and the whole organism. I am afraid that immunologists have thrown the baby out with the bathwater. Now that most of us seem to 
agree that there is more to tolerance than 'clonal deletion', it is time to revisit Jerne and consider the role of cellular and molecular networks in the establishment and maintenance of natural tolerance. This may also provide answers to other unsolved problems, for example the mechanisms controlling lymphocyte homeostasis-as each lymphocyte expresses unique V-regions, the control of their numbers must be related to the respective diversity repertoire and, therefore, of dominant selftolerance.

The other difficulty with tolerance, I would think, relates to its robustness. As indicated by experiments with knockout or transgenic mice, the acquisition of tolerance is a robust process, making it likely that a variety of mechanisms have evolved. It would be surprising therefore if the question of natural tolerance will be solved by a single experimental system, as was the case for other central questions, such as the generation of antibody diversity through gene rearrangement or the structure of the $\mathrm{MHC}$ that revealed MHC-restricted T-cell recognition. A putative solution will eventually come from a collection of laboratories, using different experimental systems and working on various aspects of the problem, which could be best achieved through a mutual cooperative project. The question of how natural tolerance develops is fundamental; the answer has an enormous clinical significance and it requires large and coordinated efforts. Perhaps immunologists should draft such an ambitious agenda and announce targets and deadlines, comparable to their colleagues in the Human Genome Project. The clinical benefits from such an undertaking are still in the future, but the collaborative spirit that has prevailed in the sequencing project, as well as its huge dimension, has increased the credibility of scientists and the general public's interest in their work. The proposal of an 'immune tolerance network' sounds like just another EC project, but it would be very different if kept among scientists devoted to understanding the basic mechanisms. And I have little doubt that, if we do solve the scientific questions, clinical progress will soon follow. The huge number of sufferers from allergies, autoimmune diseases, cancer and infectious diseases are surely reason enough to concentrate our efforts.

\section{References}

Jerne, N.K. (1974) Towards a network theory of the immune system. Ann. Immunol. (Inst. Pasteur), 125C, 373-389.

Bach, J.-F. (2002) The effect of infections on susceptibility to autoimmune and allergic diseases. N. Engl. J. Med., 347, 911-920.

Reichert, J.M. (2001) Monoclonal antibodies in the clinic. Nat. Biotechnol., 19, 819-822.

Rappuoli, R., Miller, H.I. and Falkow, S. (2002) The intangible value of vaccination. Science, 297, 937-940.

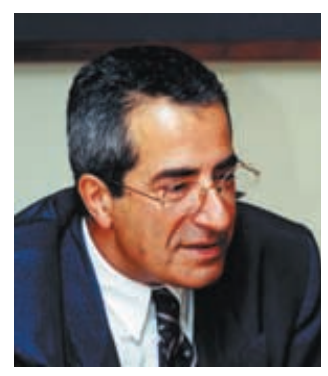

Antonio Coutinho is Director of Research at the CNRS (France) and Director of the Instituto Gulbenkian de Ciência in Portugal.

E-mail: coutinho@igc.gulbenkian.pt

DOI: 10.1093/embo-rep14orts/kvf226 